\title{
Methodological issues in economic evaluations of disease prevention and health promotion: an overview of systematic and scoping reviews
}

\author{
Yana Seleznova ${ }^{*}$ (D, Adrienne Alayli@, Stephanie Stock@ and Dirk Müller(D)
}

\begin{abstract}
Background: We aimed to provide a comprehensive overview of methodological challenges in economic evaluations of disease prevention and health promotion (DPHP)-measures.

Methods: We conducted an overview of reviews searching MEDLINE, EMBASE, NHS Economic Evaluation Database, Database of Promoting Health Effectiveness Reviews, Cochrane Database of Systematic Reviews (CDSR) and Database of Promoting Health Effectiveness Reviews (DOPHER) (from their inception to October 2021). We included both systematic and scoping reviews of economic evaluations in DPHP addressing following methodological aspects: (i) attribution of effects, (ii) outcomes, (iii) inter-sectoral (accruing to non-health sectors of society) costs and consequences and (iv) equity. Data were extracted according to the associated sub-criteria of the four methodological aspects including study design economic evaluation (e.g. model-based), type/scope of the outcomes (e.g. outcomes beyond health), perspective, cost categories related to non-health sectors of society, and consideration of equity (method of inclusion). Two reviewers independently screened all citations, full-text articles, and extracted data. A narrative synthesis without a meta-analysis or other statistical synthesis methods was conducted.
\end{abstract}

Results: The reviewing process resulted in ten systematic and one scoping review summarizing 494 health economic evaluations. A lifelong time horizon was adopted in about 23\% of DPHP evaluations, while $64 \%$ of trial-based evaluations had a time horizon up to 2 years. Preference-based outcomes (36\%) and non-health outcomes (8\%) were only applied in a minority of studies. Although the inclusion of inter-sectoral costs (i.e. costs accruing to non-health sectors of society) has increased in recent years, these were often neglected (between 6 and 23\% depending on the cost category). Consideration to equity was barely given in economic evaluations, and only addressed in six of the eleven reviews.

Conclusions: Economic evaluations of DPHP measures give only little attention to the specific methodological challenges related to this area. For future economic DPHP evaluations a tool with structured guidance should be developed.

This overview of reviews was not registered and a published protocol does not exist.

\footnotetext{
* Correspondence: yana.seleznova@uk-koeln.de

Institute for Health Economics and Clinical Epidemiology, Faculty of Medicine and University Hospital of Cologne, Gleueler Str. 176-178, 50935 Cologne, Germany
}

(c) The Author(s). 2021 Open Access This article is licensed under a Creative Commons Attribution 4.0 International License, which permits use, sharing, adaptation, distribution and reproduction in any medium or format, as long as you give appropriate credit to the original author(s) and the source, provide a link to the Creative Commons licence, and indicate if changes were made. The images or other third party material in this article are included in the article's Creative Commons licence, unless indicated otherwise in a credit line to the material. If material is not included in the article's Creative Commons licence and your intended use is not permitted by statutory regulation or exceeds the permitted use, you will need to obtain permission directly from the copyright holder. To view a copy of this licence, visit http://creativecommons.org/licenses/by/4.0/. The Creative Commons Public Domain Dedication waiver (http://creativecommons.org/publicdomain/zero/1.0/) applies to the data made available in this article, unless otherwise stated in a credit line to the data. 
Keywords: Disease prevention, Health promotion, Economic evaluation, Methods, Systematic review, Health economics

\section{Background}

In light of rapidly ageing populations, disease prevention and health promotion (DPHP) is attracting increasing attention from health policy makers all around the world [1]. According to the World Health Organization (WHO), disease prevention aims to minimize the burden of specific diseases and their associated risk factors by covering population-based and individually focused interventions. Slightly different, health promotion complements these efforts by empowering people to increase their control over determinants of health and developing supportive environments [2].

In 2015, across the member states of the Organisation for Economic Co-operation and Development (OECD), the financial resources spent on DPHP were on average less than $3 \%$ of all health spending [1]. An increase of investments in DPHP programs would require conclusive and valid information about the costs and benefits of DPHP-measures. Usually, this information is provided to funding agencies by health economic studies such as cost-effectiveness analyses (CEA, where benefits are expressed in natural effects or physical units), cost utility analyses (CUA, with benefits as health state preference scores), cost-benefit analyses (CBA, monetary terms), cost-minimization analyses (CMA, where only costs are compared) and cost-consequences analyses (CCA with an array of output measures as benefit) [3]. In addition, the social return on investment (SROI) methodology targets broader socio-economic outcomes by analysing views of multiple stakeholders and expressing these in a singular monetary ratio [4].

Although economic evidence for DPHP is increasing [5], health economic studies for DPHP can be affected by significant methodological challenges. Motivated by the Wanless report [6] that described factors likely to have an impact on the resources required to deliver a high-quality health service, the Public Health Research Consortium (PHRC) from the United Kingdom (UK) identified four key elements of economic evaluation in DPHP [7].

(i) the attribution of intervention effects should ensure an adequate reflection of a complex DPHP-measure; since trial-based designs such as randomised controlled trials (RCTs) are not always feasible, economic modelling offers a flexible approach, as it uses multiple data sources [8]. Similarly, the applied data on the consideration of (ii) outcomes and (iii) inter-sectoral costs and consequences (i.e., costs and monetary benefits which spread to other sectors) should reflect the specific context of DPHP. Furthermore, (iv) health equity is one of the main objectives stated in public health policy worldwide [9].

To achieve the social goal of allocating funding more efficiently between health care and public health, it is vital that valid and comparable analytic methods are used for both [6]. Therefore, the objective of this overview was to summarize the above listed key elements for methodological rigor in economic evaluations of DPHPmeasures. The evidence was obtained from systematic and scoping reviews of a) previous health economic analyses over a broad range of DPHP areas/measures with b) a methodological focus on the attribution of effects, outcomes, inter-sectoral costs and consequences, or equity. We also discuss the reported challenges in view of published recommendations for economic evaluations of DPHP interventions obtained from various methodological papers.

\section{Methods}

Our overview of reviews was reported according to reporting guidance in the Preferred Reporting Items for Systematic Reviews and Meta-analyses (PRISMA) 2020 reporting guideline statement [10] (see checklist in Additional file 1). Our overview of reviews was neither registered nor a protocol was published.

\section{Search strategy}

A bibliographic search was performed in MEDLINE (via Pubmed), EMBASE (via Elsevier), NHS Economic Evaluation Database (NHS EED), Database of Promoting Health Effectiveness Reviews (DOPHER) and Cochrane Database of Systematic Reviews (CDSR). All search strategies are available in Additional file 2. The websites of the OECD, the 'Medical Research Council' and the PHRC and the reference lists of the retrieved articles were also examined (Additional file 3). The search was conducted in September 2020 (updated in October 2021) and neither limits due to publication date nor language were applied.

\section{Eligibility criteria}

We searched for systematic and scoping reviews that provided data on methodological aspects of full health economic DPHP evaluations (i.e. evaluations which compare the costs and effects of two or more alternatives). Scoping reviews differ from systematic reviews in that they provide a broad overview of the evidence on a topic, while systematic reviews are used to address more 
specific questions based on particular criteria of interest (e.g., population, intervention, outcome) [11]. We included reviews which aimed specifically at the methodological aspects of economic evaluations of DPHPmeasures. Reviews were eligible if they i) evaluated full health economic studies addressing evaluations of any DPHP intervention and ii) provide a beforehand stated emphasis on the methodological assessment of the PHRC criteria [7]: (i) the attribution of effects, (ii) the selection of outcomes, (iii) the inclusion of inter-sectoral costs and consequences or, (iv) the consideration of equity aspects in the included studies. That means that, articles which did not prospectively address a methodological focus (via research questions or extraction criteria) were excluded. Reviews that targeted a specific study design (e.g., solely trial-based or model-based evaluation) or study type only (e.g., social return of investment studies), vaccination programs, genomic sequencing, or disease management programs were also excluded due to their specific nature.

\section{Study selection process}

Two reviewers (DM and YS) independently screened the search result (i.e., citations and full-texts) and extracted data from the selected studies using predefined eligibility criteria. Data from the selected studies were extracted by standardized data extraction forms. Differences were resolved through discussion.

\section{Assessment of methodological quality}

We assessed the quality of the reviews using several criteria from the Assessment of Multiple SysTemAtic Reviews tool (AMSTAR) [12], We focused on (1) the study objective, (2) the adequate provision of inclusion criteria, (3) a double-check principle for search and data extraction, (4) details on the literature search, (5) a risk of bias assessment, and (6) a discussion of potential heterogeneity (e.g. differences in modelling approaches, settings, or perspectives). These criteria were chosen because they were assumed to be of particular importance for the quality of the reviews (e.g., addressing meta-analyses). The assessment was undertaken by one reviewer (YS) and cross-checked by another reviewer (DM).

\section{Data extraction}

Based on guidance for evidence synthesis [10, 13], we focused on methodological aspects of economic evaluations targeting DPHP interventions that were likely to affect the study results. Information was selected for the PHRC criteria [7], four main methodological challenges which were derived from evaluating empirical studies of the NHS Economic Evaluation Database (NHS EED). The identified key challenges [7] correspond to a summary of suggestions from key health economics commentators (e.g.,
Edwards 2013 [14]). To emphasize on DPHP, these specific aspects were preferred to a more general quality assessment. For further assessment, various sub-criteria were selected to reflect the tendency in how researchers deal with specific methodological issues.

\section{Attribution of effects: trial versus model based-design, time horizon}

According to specific recommendations for economic analyses of DPHP the time horizon and an appropriate study design has to be chosen [15]. The time horizon of the analysis should extend far enough to reflect important differences between the strategies under evaluation. This is of particular interest for interventions where costs and benefits are falling apart (e.g., media campaigns or behavioral measures). A trial-based (e.g., a randomised-controlled trial) evaluation examines costs and outcomes within a predefined observation period; a model-based evaluation goes beyond this period and either extrapolates costs and outcomes obtained from a trial (e.g., lifetime) or syntheses data from different sources (e.g., clinical trials and routine data).

\section{Outcomes: health or preference-based outcomes, intermediate outcomes, non-health outcomes}

Intermediate outcomes are commonly used for linking the effect with final health outcomes but causation is often taken into question. For CUAs which are preferred by many decision makers, health outcomes were transferred into preference-based outcomes (e.g., qualityadjusted life years (QALYs)). Due to their complex nature, DPHP interventions may also affect wider or nonhealth outcomes, i.e. outcomes that are not covered by dimensions of the EQ-5D: mobility, self-care, usual activities, pain/discomfort and anxiety/depression [16]. On an individual level, non-health-outcomes refer to selfconfidence, insights into one's own (un) healthy behavior, and perceived life control [17] which can be used as a single measure or be included in wider conceptualizations of quality of life or wellbeing.

\section{Inter-sectoral costs and consequences: perspective and cost categories}

A major challenge in DPHP is to include inter-sectoral costs and consequences, i.e. categories of costs that accrue to non-health sectors of society and could inform CBAs. Based on Drost et al. we assessed the cost categories 'education' (e.g., learning aids), 'labor and social security' (e.g., productivity losses due to absence from work or costs due to reintegration after imprisonment), 'household and leisure' (e.g., informal care), 'criminal justice' (e.g., police interventions) and, 'other individual and family effects' (e.g., family conflicts). These costs may contribute a considerable amount to the total costs and benefits of 
interventions in the health care sector [18]. The perspective taken for an analysis determines the extent to that inter-sectoral costs and consequences will be considered. For example, an economic study of a DPHP-measure to prevent substance abuse rarely considered criminal justice costs if the study was funded by the healthcare sector [19].

\section{Equity: consideration and method of inclusion}

In response to policy concerns regarding health equity, economic evaluation of DPHP should provide useful evidence on health equity impacts. Who gains and who loses out from a health program depends individual health risks, uptake of and adherence to measures, capacity to benefit, and-crucially-who bears the opportunity costs of diverting scarce resources from other uses [20].

\section{Data synthesis}

Because the quantitative information obtainable from the reviews was limited, a narrative synthesis without a meta-analysis or other statistical methods was conducted
$[21,22]$ In particular, to summarize the information about the methodological aspects we tabulated [13] both the qualitative (e.g. scope of the analysis or types of outcomes) and the quantitative data (e.g. frequencies of corresponding outcomes) for each review included. Further, based on the reported data in the included reviews, we estimated the weighted proportion of studies meeting the sub-criteria of extracted data across the included reviews and presented the results visually using bar charts.

\section{Results}

\section{Reviews identified using the search}

The search returned 2452 records. After screening titles and abstracts, 59 records were examined for potential eligibility. The inclusion criteria assessment resulted in ten systematic and one empirical (scoping) review (Fig. 1) [5, 19, 23-31] Excluded reviews are shown in Additional file 4. Of the included reviews (Table 1), five addressed different preventive measures for older people $[25,27]$ or children/adolescents [26, 28,30], while the others targeted physical activity [23], implementation of DPHP

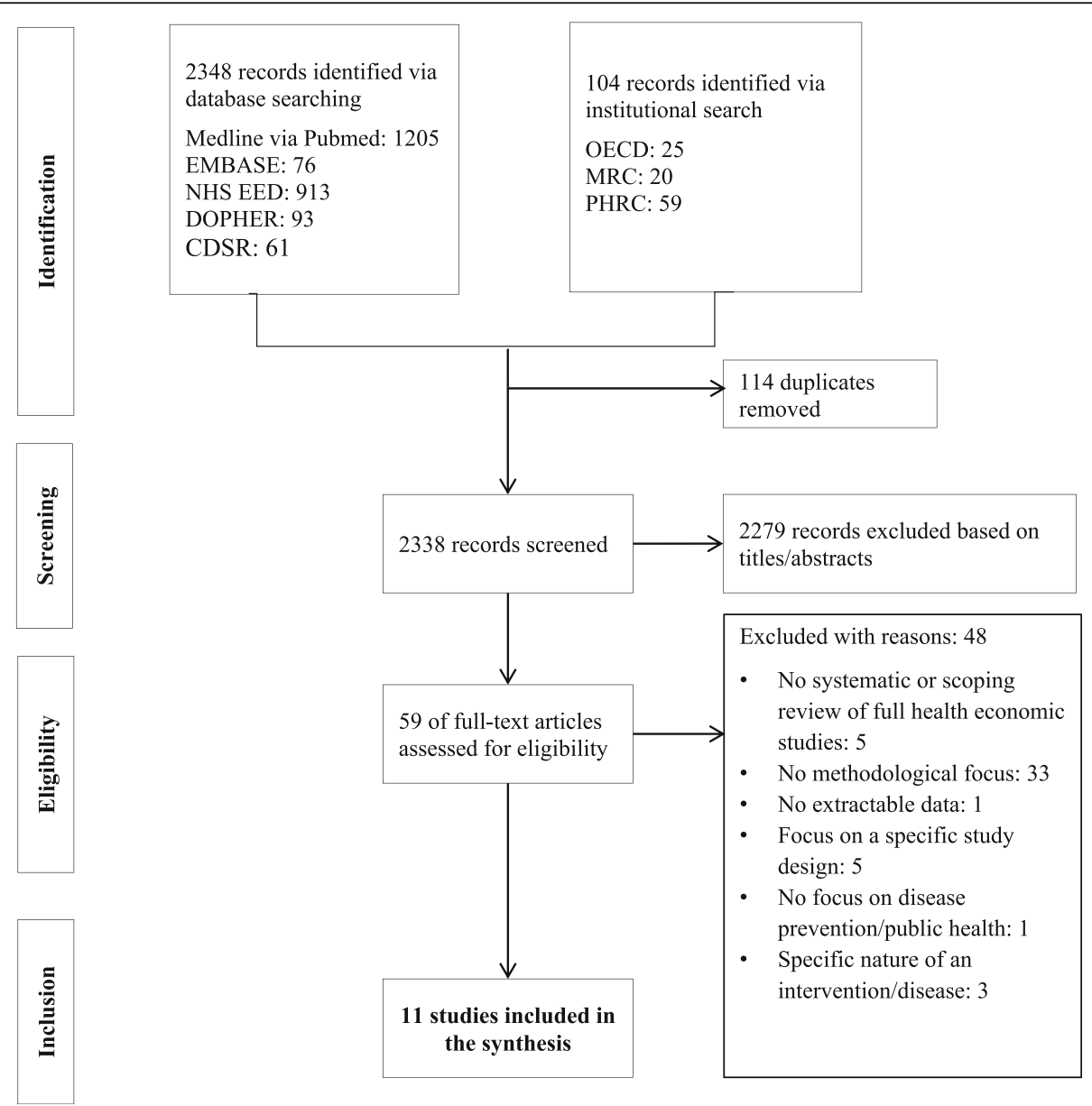

Fig. 1 Flow diagram of the search. OECD, Organisation for Economic Cooperation and Development; MRC, Medical Research Council; PHRC, Public Health Research Consortium 
Table 1 Summary of methodological aspects reported in reviews of health economic analyses

\begin{tabular}{|c|c|c|c|c|c|}
\hline Source & $\begin{array}{l}\text { Area / Study } \\
\text { type }\end{array}$ & $\begin{array}{l}\text { Attribution of } \\
\text { effects }\end{array}$ & Outcomes & Inter-sectoral costs & Equity \\
\hline $\begin{array}{l}\text { Cochrane } \\
\text { et al. (2019) } \\
\text { [23] }\end{array}$ & $\begin{array}{l}\text { Physical activity } \\
\text { and sedentary } \\
\text { behaviour } \\
\text { interventions } \\
n=15 \\
\text { CEA }=40 \% \\
\text { CUA }=33 \% \\
\text { Different types = } \\
27 \%\end{array}$ & $\begin{array}{l}\text { TBA/MBA: } \\
n=10 / 5 \\
\text { Time horizon all: } \\
-\leq 2 y: 67 \% \\
\text { - lifetime: } 33 \% \\
\text { TBA: } \leq 2 y 100 \% \\
\text { MBA: lifetime } \\
100 \%\end{array}$ & $\begin{array}{l}\text { Intermediate HO: 53\% } \\
\text { HO/PbO: } 60 \% \text { (QALYs) } \\
\text { Non-HO: } 13 \% \text { (e.g., absenteeism) }\end{array}$ & $\begin{array}{l}\text { Categories: } \\
\text { - labor/soc. security: } 20 \% \\
\text { Perspective: } \\
\text { - societal: } 27 \% \\
\text { - health sector: } 47 \% \\
\text { - not stated: } 13 \%\end{array}$ & $\begin{array}{l}\text { Implicitly included via subgroup } \\
\text { analyses: } 93 \% \\
\text { (e.g., targeting the intervention } \\
\text { to individuals in need of care) }\end{array}$ \\
\hline $\begin{array}{l}\text { Reeves } \\
\text { et al. (2019) } \\
\text { [24] }\end{array}$ & $\begin{array}{l}\text { Implementation } \\
\text { of DPHP- } \\
\text { interventions } \\
n=14 \\
\text { CEA }=86 \% \\
\text { CUA }=7 \% \\
\text { CBA }=7 \%\end{array}$ & $\begin{array}{l}\text { TBA/MBA: } \\
n=12 / 2 \\
\text { Time horizon all: } \\
-\leq 2 y: 43 \% \\
\text { - lifetime: } 0 \% \\
\text { - not reported: } \\
36 \% \\
\text { TBA: } \leq 2 y 50 \% \\
\text { MBA: lifetime } 0 \%\end{array}$ & $\begin{array}{l}\text { Intermediate HO: } 93 \% \\
\text { HO/PbO: } 29 \% \text { (QALYS, life saved) } \\
\text { Non-HO: } 7 \% \text { (violent crimes) }\end{array}$ & $\begin{array}{l}\text { Categories: } \\
\text { - education: } 43 \% \\
\text { - labor/soc. security: } 29 \% \\
\text { - criminal justice: } 7 \% \\
\text { Perspective: } \\
\text { - societal: } 42 \% \\
\text { - not stated: } 21 \%\end{array}$ & n.a. \\
\hline $\begin{array}{l}\text { Zanganeh } \\
\text { et al. (2019) } \\
\text { [30] }\end{array}$ & $\begin{array}{l}\text { Childhood and } \\
\text { adolescent } \\
\text { obesity } \\
\text { interventions } \\
n=28 \\
\text { CEA }=36 \% \\
\text { CUA }=32 \% \\
\text { CCA }=4 \% \\
\text { Different types = } \\
29 \%\end{array}$ & $\begin{array}{l}\text { TBA/MBA: } n=9 / \\
19 \\
\text { Time horizon all: } \\
\text { - } \leq 2 \mathrm{y}: 21 \% \\
\text { - lifetime: } 39 \% \\
\text { - not reported: } \\
\quad 7 \% \\
\text { TBA: } \leq 2 \mathrm{y} 67 \% \\
\text { MBA: lifetime } \\
\text { 58\% }\end{array}$ & $\begin{array}{l}\text { Intermediate HO: all studies (64\%: BMI) } \\
\text { HO/PbO: 59\% (QALYS, DALYS) } \\
\text { Non-HO: n.a. }\end{array}$ & $\begin{array}{l}\text { Categories: } \\
\text { - education: } 4 \% \\
\text { - labor/soc. security: } 57 \% \\
\text { - other: } 18 \% \\
\text { Perspective: } \\
\text { - societal: } 84 \% \\
\text { - health sector: } 8 \% \\
\text { - not stated: } 8 \%\end{array}$ & n.a. \\
\hline $\begin{array}{l}\text { Huter et al. } \\
(2018)[25]^{\mathrm{a}}\end{array}$ & $\begin{array}{l}\text { DPHP- } \\
\text { interventions for } \\
\text { older people } \\
n=8 \\
\text { CEA }=25 \% \\
\text { CUA }=25 \% \\
\text { Different types = } \\
50 \%\end{array}$ & $\begin{array}{l}\text { TBA/MBA: } n=4 / \\
4 \\
\text { Time horizon all: } \\
-\leq 2 \mathrm{y}: 75 \% \\
\text { - lifetime: } 13 \% \\
\text { TBA: } \leq 2 \mathrm{y} 100 \% \\
\text { MBA: lifetime } \\
25 \%\end{array}$ & $\begin{array}{l}\text { Intermediate HO: all studies (e.g., falls, } \\
\text { physical activity) } \\
\text { HOOPbO: } 88 \% \\
\text { (fall-related fractures, QALYs) } \\
\text { Non-HO: } 13 \% \\
\text { (social benefits such as 'general self-efficacy', } \\
\text { 'well-being', or 'loneliness') }\end{array}$ & $\begin{array}{l}\text { Categories: } \\
\text { - labor/soc. security: n.a. } \\
\text { - household/leisure: n.a. } \\
\text { - other: } 13 \% \\
\text { Perspective: } \\
\text { - societal: } 13 \% \\
\text { - not stated: } 88 \%\end{array}$ & $\begin{array}{l}\text { No adjustment for the } \\
\text { preference structure of elderly in } \\
\text { CUAs }\end{array}$ \\
\hline $\begin{array}{l}\text { Oosterhoff } \\
\text { et al. } \\
\text { (2018) [26] }\end{array}$ & $\begin{array}{l}\text { School-based } \\
\text { lifestyle } \\
\text { interventions } \\
n=23 \\
\text { CEA }=48 \% \\
\text { CUA }=17 \% \\
\text { CBA }=9 \% \\
\text { SROI }=4 \% \\
\text { Different types = } \\
22 \%\end{array}$ & $\begin{array}{l}\text { TBA/MBA: } n=9 / \\
14 \\
\text { Time horizon all: } \\
\text { - } \leq 1 \mathrm{y}: 30 \% \\
\text { - lifetime: } 43 \% \\
\text { TBA: } \leq 1 \mathrm{y} 78 \% \\
\text { MBA: lifetime } \\
71 \%\end{array}$ & $\begin{array}{l}\text { Intermediate HO: } 48 \% \text { (weight-related } \\
\text { measures or BMI) } \\
\text { HO/PbO: } 39 \% \\
\text { (QALYs or DALYs) } \\
\text { Non-HO: } 13 \% \\
\text { (school behaviour, wellbeing, outcomes to } \\
\text { the household and leisure sector, } \\
\text { environmental impacts and externalities, } \\
\text { only in CBAs, SROI) }\end{array}$ & $\begin{array}{l}\text { Categories: } \\
\text { - education: } 9 \% \\
\text { - labor/soc. security: 17\% } \\
\text { - household/leisure: } 13 \% \\
\text { - other: } 4 \% \\
\text { Perspective: } \\
\text { - societal: } 74 \% \\
\text { - healthcare: } 13 \% \\
\text { - programs perspective: 4\% } \\
\text { - not stated: } 9 \%\end{array}$ & n.a. \\
\hline $\begin{array}{l}\text { Dubas- } \\
\text { Jakóbczyk } \\
\text { et al. (2017) } \\
\text { [27] }\end{array}$ & $\begin{array}{l}\text { DPHP- } \\
\text { interventions for } \\
\text { older people } \\
N=29 \\
\text { CCA }=7 \% \\
\text { CEA }=45 \% \\
\text { CUA }=10 \% \\
\text { CBA }=10 \% \\
\text { Different types = } \\
28 \%\end{array}$ & $\begin{array}{l}\text { TBA/MBA: } n= \\
19 / 10 \\
\text { Time horizon all: } \\
-\leq 1 \mathrm{y}: 50 \% \\
\text { - lifetime: } 17 \% \\
\text { TBA: } \leq 1 \mathrm{y} \text { n.s } \\
\text { MBA: lifetime n.s }\end{array}$ & $\begin{array}{l}\text { Intermediate HO: } 35 \% \\
\text { (falls, number of falls or fallers prevented) } \\
\text { HO/PbO: } 65 \% \\
\text { (changes in SF-36 physical functioning, LYG, } \\
\text { daily functioning changes, QALYs) } \\
\text { Non HO: } 3 \% \text { (e.g., social network) }\end{array}$ & $\begin{array}{l}\text { Categories: } \\
\text { - labor/soc. security: 3\% } \\
\text { - household/leisure: } 21 \% \\
\text { - other: } 7 \% \\
\text { Perspective: } \\
\text { - societal: } 41 \% \\
\text { - payer: } 52 \% \\
\text { - unclear: } 7 \%\end{array}$ & $\begin{array}{l}\text { Lack of economic evaluations for } \\
\text { the prevention of mental health } \\
\text { problems among the population } \\
65+\end{array}$ \\
\hline $\begin{array}{l}\text { Hill et al. } \\
\text { (2017) [31] }\end{array}$ & $\begin{array}{l}\text { Alcohol } \\
\text { prevention } \\
n=27 \\
\text { CEA }=22 \% \\
\text { CUA }=48 \% \\
\text { CBA }=4 \% \\
\text { CCA }=4 \% \\
\text { SROI }=4 \% \\
\text { Different types = } \\
19 \%\end{array}$ & $\begin{array}{l}\text { TBA/MBA: } n=8 / \\
19 \\
\text { Time horizon all: } \\
-\leq 1 \text { y: } 19 \% \\
-1-5 y: 26 \% \\
->30 y \text { to lifetime: } \\
37 \% \\
- \text { not reported: } \\
11 \% \\
\text { TBA: }-\leq 1 y 50 \% \\
\text { MBA: - >30y to } \\
\text { lifetime } 53 \%\end{array}$ & $\begin{array}{l}\text { Intermediate HO: n.a } \\
\text { HO/PbO: } 67 \% \text { (e.g., QALYs) } \\
\text { Non HO: } 0 \%\end{array}$ & $\begin{array}{l}\text { Categories: } \\
\text { - education: 11\% } \\
\text { - labor/soc. security: 26\% } \\
\text { - household/leisure: } 15 \% \\
\text { - criminal justice: } 33 \% \\
\text { Perspective: } \\
\text { - societal: } 52 \% \\
\text { - health sector: } 41 \% \\
\text { - other or not stated: } 7 \%\end{array}$ & $\begin{array}{l}\text { Subgroup analysis for gender, } \\
\text { age and alcohol intake }\end{array}$ \\
\hline $\begin{array}{l}\text { Döring } \\
\text { et al. (2016) } \\
\text { [28] }\end{array}$ & $\begin{array}{l}\text { Obesity } \\
\text { prevention in } \\
\text { early childhood } \\
N=6\end{array}$ & $\begin{array}{l}\text { TBA/MBA: } n=2 / \\
4 \\
\text { Time horizon all: } \\
-\leq 2 \mathrm{y}: 33 \%\end{array}$ & $\begin{array}{l}\text { Intermediate HO: } 100 \% \\
\text { (BMl, weight, behavioral changes) } \\
\text { HO/PbO: } 33 \% \\
\text { Non HO: n.a. }\end{array}$ & $\begin{array}{l}\text { Categories: } \\
\text { - labor/soc. security: } 67 \% \\
\text { Perspective: } \\
\text { - societal: } 50 \%\end{array}$ & n.a. \\
\hline
\end{tabular}


Table 1 Summary of methodological aspects reported in reviews of health economic analyses (Continued)

\begin{tabular}{|c|c|c|c|c|c|}
\hline Source & $\begin{array}{l}\text { Area / Study } \\
\text { type }\end{array}$ & $\begin{array}{l}\text { Attribution of } \\
\text { effects }\end{array}$ & Outcomes & Inter-sectoral costs & Equity \\
\hline & $\begin{array}{l}C C A=17 \% \\
\text { CEA }=83 \%\end{array}$ & $\begin{array}{l}\text { - lifetime: } 67 \% \\
\text { TBA: } \leq 2 y \text { y } 100 \% \\
\text { MBA: lifetime } \\
100 \%\end{array}$ & & - other: $17 \%$ & \\
\hline $\begin{array}{l}\text { Alayli- } \\
\text { Goebbels } \\
\text { et al. (2014) } \\
\text { [5] }\end{array}$ & $\begin{array}{l}\text { Behavior change } \\
\text { interventions } \\
N=142 \\
C C A=8 \% \\
\text { CEA }=48 \% \\
\text { CUA }=11 \% \\
C M A=3 \% \\
\text { CBA }=6 \% \\
\text { Different types = } \\
25 \%\end{array}$ & $\begin{array}{l}\text { TBA/MBA: } n= \\
58 / 56^{\mathrm{b}} \\
\text { Time horizon all: } \\
-\leq 2 \mathrm{y}: 37 \% \\
\text {-lifetime: } 21 \% \\
\text { - not reported: } \\
22 \% \\
\text { TBA: } \leq 2 \mathrm{y} 83 \% \\
\text { MBA: lifetime in } \\
38 \% \text { (30/79 } \\
\text { modelling and } \\
\text { combined) }\end{array}$ & $\begin{array}{l}\text { Intermediate HO: } 61 \% \text { : } \\
\text { (behavior change, biomedical health } \\
\text { indicators) } \\
\text { HO/PbO: } 52 \% \\
\text { (e.g., survival, HRQOL) } \\
\text { Non HO: } 9 \% \\
\text { (e.g., increased health knowledge) }\end{array}$ & $\begin{array}{l}\text { Categories: } \\
\text { - summarized: } 8 \% \\
\text { (included costs of car accidents, } \\
\text { violent crimes, personal injury, } \\
\text { property damage, fire destruction, } \\
\text { law enforcement, and costs to } \\
\text { industry, commerce and the } \\
\text { voluntary sectors) } \\
\text { Perspective: } \\
\text { - not stated: } 43 \%\end{array}$ & Explicitly considered by no study \\
\hline $\begin{array}{l}\text { Polinder } \\
\text { et al.(2012) } \\
\text { [29] }\end{array}$ & $\begin{array}{l}\text { Injury prevention } \\
N=48 \\
\text { CEA }=38 \% \\
\text { CUA }=10 \% \\
\text { CBA }=35 \% \\
\text { Different types = } \\
17 \%\end{array}$ & $\begin{array}{l}\text { TBA/MBA: } n= \\
24 / 24 \\
\text { Time horizon all: } \\
\text { - } \leq 5 \mathrm{y}: 33 \% \\
\text { - lifetime: } 8 \% \\
\text { - not reported: } \\
\quad 17 \% \\
\text { TBA: }<2 y 17 \% \\
\text { MBA: lifetime } \\
16 \%\end{array}$ & $\begin{array}{l}\text { Intermediate HO: } 10 \% \\
\text { (falls prevented) } \\
\text { HO/PbO: } 52 \% \\
\text { (injuries prevented, life saved, QALYs) } \\
\text { Non HO: n.a. }\end{array}$ & $\begin{array}{l}\text { Categories: } \\
\text { - labor/soc. security: } 23 \% \\
\text { - household/leisure: } 2 \% \\
\text { Perspective: } \\
\text { - societal: } 69 \% \\
\text { - health care: } 25 \%\end{array}$ & n.a. \\
\hline $\begin{array}{l}\text { Weatherly } \\
\text { et al. (2009) } \\
\text { [19] }\end{array}$ & $\begin{array}{l}\text { DPHP- } \\
\text { interventions in } \\
\text { eleven public } \\
\text { health areas } \\
N=154 \\
\text { CCA }=37 \% \\
\text { CEA }=36 \% \\
\text { CUA }=27 \% \\
\text { CMA }=0 \% \\
\text { CBA }=0 \%\end{array}$ & $\begin{array}{l}\text { TBA/MBA: } n= \\
106 / 48 \\
\text { Time horizon all: } \\
\text { n.s. }\end{array}$ & $\begin{array}{l}\text { Intermediate HO: n.S. } \\
\text { (falls prevented, pounds lost) } \\
\text { HO/PbO: } 27 \% \\
\text { (QALYs, DALYs) } \\
\text { Non HO: } n \text {. S. } \\
\text { (quality of wellbeing, public preferences for } \\
\text { the (dis) benefits of a water fluoridation } \\
\text { program) }\end{array}$ & $\begin{array}{l}\text { Categories: } \\
\text { - education: } 3 \% \\
\text { - labor/soc. security: 20\% } \\
\text { - household/leisure: } 2 \% \\
\text { - criminal justice: } 4 \% \\
\text { - other: } 16 \% \\
\text { Perspective: } \\
\text { - payer: } 32 \% \\
\text { - societal: } 31 \% \\
\text { - not stated: } 24 \%\end{array}$ & $\begin{array}{l}\text { QALYs were not explicitly equity- } \\
\text { weighted, some studies con- } \\
\text { ducted equity-related sub- } \\
\text { groups analyses }\end{array}$ \\
\hline
\end{tabular}

$B M I$ body mass index, CCA cost-consequences analysis, CBA cost-benefit analysis, CEA cost-effectiveness analysis, CMA cost minimization analysis, CUA cost-utility analysis, $E E$ economic evaluation, $H O$ health outcome, $N$ number, n.a not addressed, n.s proportion or details not specified, $M B A$ model-based analysis, $P b O$ Preference-based outcome, $T B A=$ trial-based analysis, $Q A L Y$ quality-adjusted life years, $D A L Y S$ disability-adjusted life years, $H R Q O L$ health-related quality of life, SROI social return on investment, WTP willingness to pay, $y$ year(s)

${ }^{a}$ Eight of 37 studies included because other studies were already included in Dubas-Jakóbczyk et al. [27]

${ }^{b}$ Remaining studies were based on a combination of TBA and MBA or, the design was not described (3.5\%)

[24], alcohol prevention [31], behavior change [5], injury prevention [29], or different areas of prevention [19]. Overall, these reviews included 494 economic analyses (CEA: 42\%, CUA: 20\%, different types of economic study: 16\%, CCA: 15\%, CBA: 6\%, CMA: 1\%. SROI: 0,4\%).

\section{Attribution of effects}

In $44 \%$ of the individual studies the time horizon was less than 5 years, while a lifelong time horizon was adopted in about $23 \%$ of the analyses. Trial-based approaches were used more often than model-based approaches to evaluate DPHP interventions (56\% vs. $44 \%$ ), while efforts to synthesize trial data with a modelling approach were found in 10\% of DPHP evaluations. With regard to trial-based analyses, $64 \%$ had a time horizon up to 2 years [5, 23-26, 28-31].

Overall, the research designs varied widely and were insufficiently reported. For example, comparators under evaluation were not described clearly $[19,26]$, costs and effects were often not discounted to adjust for different timing [5, 29] or - in model-based studies - the model choice or the underpinning assumptions (e.g., maintenance of effects over time) were not explained clearly or justified sufficiently [23, 28, 30]. Similarly, heterogeneous methods were used in both model and trial-based studies to extrapolate intermediate to final outcomes (e.g., change in BMI and its influence on morbidity), to extend the timeframe of the analysis (e.g., extrapolating the impact on future health-related outcomes), and/or to synthesize data from different sources (e.g., decision-analytic model or regression model) $[19,26]$.

\section{Outcomes}

In $56 \%$ of individual studies, researchers relied on intermediate health outcomes such as biomedical health indicators (e.g., weight loss) [5, 19], falls as an intermediate for fractures [19, 27], or indicators related to the avoidance of health service use (e.g., hospital admissions prevented) [5, 27]. Many studies with a long-time horizon 
did not account for the possibility of diminishing intervention effects [26].

Whereas measures of (direct) health outcomes such as life years gained were used rarely, preference-based outcomes were applied in about $36 \%$ of the individual studies. Preferences were expressed by using QALYs or disabilityadjusted life years (DALYs) and, by converting a statistical life into monetary values resulting in a CBA. Moreover, contingent valuation willingness-to-pay (WTP) surveys were used for eliciting public preferences $[7,19]$.

Eight percent of the economic studies measured nonhealth outcomes or used broader definitions of quality of life or wellbeing (e.g., measures of health literacy, improvement of self-management capacities, and social networks). For example, two studies (1.4\%) used measures of global quality of life or wellbeing in evaluations of behavior change interventions [5]. Furthermore, outcomes for individuals not directly targeted by the intervention and community-level outcomes were rarely incorporated when evaluating an intervention $[5,26]$.

\section{Inter-sectoral costs and consequences}

The costs of labor/social security (i.e., productivity losses due to absence from work) were considered in $23 \%$ of the individual studies. In contrast, the costs of education (7\%), household \& leisure (6\%) and criminal justice (8\%) were included only in a small number of studies. Other monetary individual/family effects were included in $14 \%$ of the studies. Compared to reviews published before 2017 [5, 19, 28, 29], in more recent reviews there was a slight increase in the consideration of inter-sectoral costs and consequences.

The reviews raised concerns about an often incomplete reporting of both health sectoral costs and intersectoral costs. Moreover, several reviews addressed inconsistencies in the cost analysis process, such as the usage of different approaches to classify, measure and value the same cost categories [5, 24, 25, 27].

According to the included reviews, the underlying economic studies showed often inconsistencies between the chosen perspective and the inter-sectoral costs considered. For example, although $46 \%$ of all studies applied a societal perspective, the costs due to labor/social security were calculated for only half of these studies. Furthermore, many studies failed to state the perspective of the analysis [5, 19, 23, 24]. In some studies, the perspective was indicated without specifying the cost categories considered [26] or the range of costs and consequences was narrower than expected for the stated perspective [23].

\section{Equity}

While concerns of equity were not addressed in almost half of the reviews $[24,26,28-30]$, the authors of five reviews concluded that equity considerations were usually disregarded in economic studies [5, 19, 25, 27, 31]. In particular, Alayli-Goebbels et al. (2014) [5] noted that the impact on equity was not examined at all, neither by using equity-weighted utilities nor by calculating costeffectiveness ratios for different socio-economic groups. Although some studies provided calculations for subgroups that might be relevant for equity considerations, these studies usually summarized utilities, implying equal weighting no matter to whom the benefits accrue [19]. Only the most recent review noted that almost all of the included analyses considered equity. However, the findings in this review suggest that analysts are not performing equity analyses in a comprehensive or consistent manner [23]. For example, only one of 15 studies in this review researched socio-economic status by asking

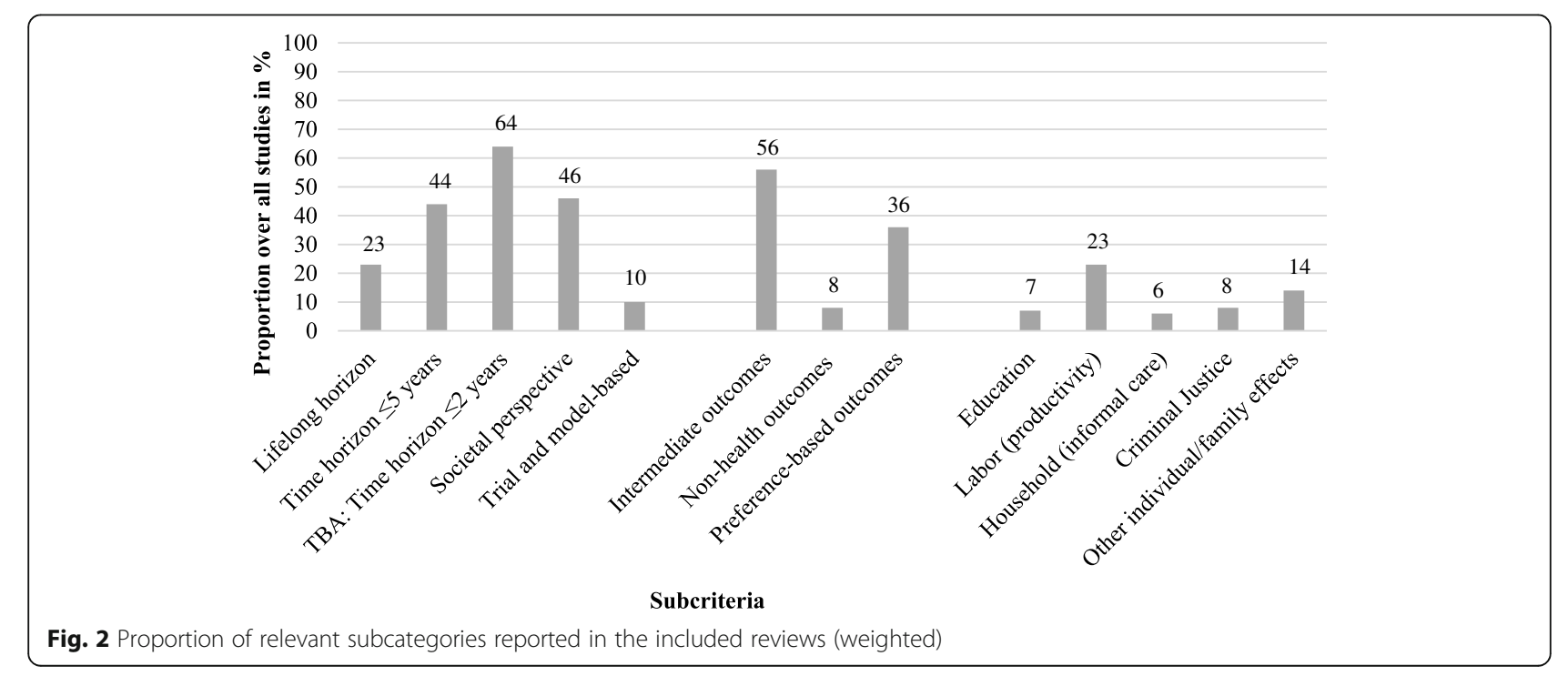


participants about their WTP for an intervention component.

Figure 2 provides an overview of the overarching analyses conducted to summarize evidence on methodological approaches in the underlying studies for each of the four PHRC criteria (Fig. 2 and Additional file 6)

\section{Methodological quality of the reviews}

Based on several criteria of the AMSTAR-tool, the methodological quality of the reviews was sufficient [12]. All reviews provided information on their objective, inclusion criteria, literature search, and study and data extraction methods. The quality of the underlying economic studies was addressed in 9 reviews [5, 23-30] while heterogeneity between the included analyses was addressed in seven [24-30]. Reeves (2019) [24] and Alayli-Goebbels (2014) [5] explicitly evaluated the methodological quality of the underlying studies. Moreover, Reeves (2019) [24] differentiated between the methodological and reporting quality of the included economic analyses (see Additional file 5).

\section{Discussion}

Our comprehensive overview summarizes how economic evaluations of DPHP-measures considered methodological challenges of economic evaluation in DPHP. According to eleven reviews (494 analyses), there were methodological inconsistencies over a broad range of DPHP measures for all predefined dimensions (attribution of effects, quantification of outcomes and costs, consideration of equity). Whereas current theoretical debates (e.g., [9, 14, 18, 32, 33]) of specific challenges in economic evaluations of DPHP are rarely reflected in health evaluation practice, non-compliance with wellestablished general standards of economic evaluation [34] was also often observed. As a result, the information obtained from many health economic analyses of DPHP measures may be limited in value for decision maker. Encouragingly, recent reviews $[23,24,30]$ indicate a tendency to address the specific challenges of DPHP more appropriately (e.g., inter-sectoral costs and consequences, equity).

To account for the attribution of effects, the increased number of relationships and interactions in DPHPmodels (e.g., between behavior changes, biomedical health indicators, patients, care giver) requires the application of models with more flexibility than conventional Markov-models (e.g., [5, 25, 28, 31]). Because the costeffectiveness of a DPHP measure is predominantly determined by individual behavior, some authors proposed more sophisticated modelling techniques such as econometric modeling, individual-level Markov models, discrete event simulation (DES), social network analysis or agent-based simulation (ABS) [5, 19].
More specifically, a conceptual modelling approach as recommended by Squires et al. 2016 can support the development of model structures that reflect the dynamic and complex nature of DPHP interventions [32]. Conceptual modelling allows to consider data on the uptake of an intervention, unforeseen participant responses (e.g., non-participation), or variations in the provision of measures (e.g., frequency or the care-giver involved). To reflect a complex and dynamic DPHP-measure, conceptual modeling framework refers to a holistic way of thinking about the interactions between parts within a system and with its environment [35]. By defining multiple system levels which are subjectively defined, higher level systems correspond to lower level systems reflecting more detailed aspects [32].

Moreover, conceptual models are assumed to allow the consideration of various determinants of health [36] (e.g., the social, economic, and physical environment, as well as a person's individual characteristics). By including broader determinants of health, conceptual models can also be used to facilitate identification of non-health costs and outcomes associated with the DPHP-measures [32].

When preferring a trial-based approach, clusterrandomised community trials or registry-based RCTs (i.e., pragmatic trials that use registries for data collection, randomisation, and follow-up) may prove an appropriate tool for comparative effectiveness in real-world settings [37]. However, the majority of trial-based evaluations are based on short time horizons (e.g. [23]), indicating that decision analytic modelling should be used more often (to date: $10 \%$ ) for extrapolating the findings of a study beyond the period of observation (e.g., $[5,23,26])$.

With regard to the outcomes used for evaluations of DPHP, authors of the reviews reported both a lack of usage of non-health outcomes and a lack of valuing outcomes. According to the reviews, intermediate outcomes were predominantly used in health economic analyses of DPHP interventions (56\%), while non-health outcomes and preference-based outcomes are rarely applied (8 and $36 \%$, respectively), resulting in a broad consensus for increasing their consideration [5, 25-27]. Based on current methodological guidance, some researchers argue for the inclusion of non-health outcomes in a tool [14, 38, 39] but disagree on what is more promising: the development of a new tool, or the use of established questionnaires with a theoretical founding (e.g., QALYs).

Over $60 \%$ of the studies in the included reviews did not attempt any outcome valuation, i.e. they provide a CCA $(15 \%)$ or CEA (42\%). In contrast, $20 \%$ of the studies were preference-based CUAs, with valuations restricted to health outcomes expressed in QALYs or DALYs. A CUA is grounded in a non-welfarist approach, while other studies with outcome valuation were based on a CBA (6\%), a welfarism-approach with outcomes 
valuated in monetary terms using willingness-to-pay estimates or SROI. A CBA might be useful for adapting the analysis to the relevant outcomes beyond health [31]; however, practical issues associated with monetary valuation remain unresolved [32]. Given the ongoing discussion on how to measure and value non-health outcomes, a CCA appears to be useful for providing a disaggregated overview of DPHP interventions [19, 32].

For the future, available methods should increasingly be used for the valuation of non-health outcomes. These include 'contingent valuation' [5], 'willingness to pay' for eliciting a benefit of an intervention [5, 19], and 'discrete choice techniques' [40]. In addition, outcomes caused by externalities (i.e. outcomes for individuals who are not directly targeted by the intervention) should be included more often [5].

Because inter-sectoral costs and consequences were excluded in economic studies of DPHP for a long time [5, 19, 28, 29], several researchers highlighted the need to consider these costs $[5,14,19,32,41]$. However, the tools developed were heterogeneous and showed limited evidence on validity and reliability [42].

In 2013, Drost et al. developed and applied several approaches for the inclusion of impacts for the education and criminal justice sectors [18], resulting in a slight increase of reporting inter-sectoral costs and consequences in later reviews. However, a prerequisite is that data valuation can be based on proxy unit prices, on selfconstructed unit prices or on labor costs [18].

The consideration of labor costs, even in evaluations adopting a societal perspective, is controversial for different reasons. First, the inclusion of labor costs is contentious and, there is no consensus or guidance on how to measure and value productivity appropriately, in particular with regard to unpaid work. Second, productivity costs are often disregarded because they are assumed to be negligible. However, the exclusion of productivity costs may result in underestimating the cost-effectiveness of DPHP measures [25].

DPHP interventions can be evaluated from a number of different perspectives, e.g., the health sector perspective, the public sector perspective or the perspective of particular agencies involved in the system [32]. The failure to consistently adopt a societal perspective $(46 \%$ and, in studies with a societal perspective, the omission of certain relevant costs such as those relating to productivity losses and participants' time, may underestimate the cost-effectiveness of DPHP-measures [43] and often precludes a deeper understanding of the monetary consequences of DPHP.

Although validated and well-accepted tools for the inclusion of these inter-sectoral costs are lacking, the adoption and consistent application of a societal perspective would stimulate efforts to include costs and effects beyond the health sector [42].

Although many authors have called for the incorporation of equity concerns in economic evaluations in DPHP, existing methods $[9,38,44]$ are neither common practice nor included in guidelines for economic evaluations. As a result, most evaluations in DPHP fail to consider equity. At least, the most recent review observed an increased consideration of equity aspects by conducting subgroup analyses or targeting a population deemed in need of intervention [23]. This might indicate growing awareness of this issue.

In general, equity considerations might be qualitatively examined by providing background information on aspects of fairness $[5,19,45]$. A more extensive approach aims to calculate cost-effectiveness for different 'equityrelevant' subgroups [9] characterized by socio-economic status, geographical location or ethnicity [5, 19, 23, 45]. A third approach aims to estimate the opportunity cost of a particular equity consideration in terms of population health sacrifice. For example, the life years lost by pursuing a more equitable option can be opposed to a more health maximizing option. The resulting opportunity cost is an approximate for the monetary value of considering equity $[9,20]$.

The equity weighting analysis approach requires more information. This method aims to explicitly value the reduction of health inequality $[19,45]$. This analysis quantifies the trade-offs between improving total health and other equity objectives. The idea of this approach is to weight health gains (e.g., life years) with different equityrelevant characteristics. The weights are based on values elicited from specific stakeholders such as the general public or/and policy makers. The weights can be elicited using common health outcome valuation techniques (e.g., discrete choice experiments) [9, 20] Weatherly et al. (2009) emphasize that more research is needed on equity-weighting issues for an individual's socialeconomic status, personal responsibility for health risks, and the preference toward treating current illnesses versus preventing future health risk [19]. Alternatively, equity might be considered by Sen's capability theory [46] which accounts for the distribution of capability across society.

\section{Strengths and limitations of this overview}

By taking up the quality criteria for economic evaluations identified by the PHRC, specific methodological challenges could be observed for predefined key elements of DPHP-interventions. Based on these categories, the results of our overview provide a comprehensive picture of the challenges for economic evaluations in DPHP. Thus, this analysis may provide a starting point 
for developing structured guidance for conducting economic evaluations in DPHP.

Although the methods used for this analysis were in line with the principles of good systematic reviewing [10, 12, 13] some limitations are inherent: first, the overview was limited to studies focusing on methodological aspects. As a result, we did not include methodological challenges from systematic or scoping reviews of economic studies without this focus.

Second, in the reviews methodological aspects were heterogeneously reported. Furthermore, data on subcriteria to be extracted was not always provided (or extractable), resulting in only approximate estimates and the impossibility to evaluate interrelationships between these sub-criteria (e.g., to calculate the proportion of studies with a societal perspective that included productivity costs). Therefore, this overview presents a trend in the modus operandi for DPHP evaluations.

Third, there may be other dimensions and subdimensions reflecting relevant aspects of the validity of economic DPHP evaluations which were not addressed in the PHCR-criteria (and thus not in our overview). Among others, these may include dealing with uncertainty, the choice of discount rates, and the inclusion of costs due to screening or costs resulting from added life years. However, by relying on the PHCR-criteria, we focused on dimensions of quality that were considered to be essential in the context of DPHP [7].

Fourth, the specific relevance of each of the challenges (i) to (iv) in an economic study depend on several factors such as the specific interventions under evaluation, the target group, the objective, the chosen study type or the perspective. As a result, the need to make particular efforts in the methodological key elements may have differed between the studies. Because we included evaluations of any DPHP-measure, the differences requiring to address the specific challenges more or less were not considered in this overview.

Finally, some economic evaluations might have been included in different reviews. Since not all reviews reported the individual studies they included, we cannot rule out that double-counting occurred (Fig. 2). However, we believe that the main conclusions drawn from this analysis would not alter.

\section{Conclusion}

Economic evaluations of DPHP measures often disregard the specific methodological challenges related to this area. Future research should aim to develop structured guidance (including a checklist) that provides recommendations for researchers who intend to economically evaluate a DPHP measure. Ideally, this tool should combine the basic principles of health economic evaluation with the particular challenges relating to DPHP. For this purpose, we recommend establishing an expert task force to identify (i) which aspects of already established guidelines may correspond to DPHP and, (ii) which issues require further specifications or methodological advancement. Additionally, there is a strong need for an increased dialogue between different stakeholders (e.g. decision-makers and academia) in order to scrutinize the boundaries of economic analyses in the field of DPHP.

\section{Abbreviations}

AMSTAR: Assessment of Multiple SysTemAtic Reviews; ABS: Agent-based simulation; CBA: Cost-benefit analysis; CCA: Cost-consequences analysis; CDSR: Cochrane Database of Systematic Reviews; CEA: Cost-effectiveness analysis; CHAP: Cardiovascular Health Awareness Program; CMA: Costminimization analysis; CUA: Cost-utility analysis; DALY: Disability-adjusted Life Year; DES: Discrete Event Simulation; DPHP: Disease Prevention and Health Promotion; DOPHER: Database of Promoting Health Effectiveness Reviews; ICECAP-A: Investigating Choice Experiments Capability Measure for Adults; NNS EED: NHS Economic Evaluation Database; OECD: Organisation for Economic Co-operation and Development; PHRC: Public Health Research Consortium; QALY: Quality-adjusted Life Year; RCT: Randomised controlled trial; SROI: Social return on investment; UK: United Kingdom; WTP: Willingness-to-pay

\section{Supplementary Information}

The online version contains supplementary material available at https://doi. org/10.1186/s12889-021-12174-w.

Additional file 1. PRISMA 2020 Checklist.

Additional file 2. Electronic Database Searches.

Additional file 3. Institutions.

Additional file 4. List of the excluded studies.

Additional file 5. Assessment of methodological aspects of the reviews included.

Additional file 6. Quantitative Synthesis.

\section{Acknowledgements}

Not applicable.

\section{Authors' contributions}

All authors were involved in the conception and design of this review. Selection of articles, data extraction and analysis were done by YS and DM. YS and DM guided the data extraction and analysis. All authors contributed to data interpretation. YS, DM and AA participated in writing the final manuscript. SS reviewed the final manuscript. All authors read and approved the final manuscript.

\section{Funding}

This research did not receive any specific grant from funding agencies in the public, commercial, or not-for-profit sectors. Open Access funding enabled and organized by Projekt DEAL.

Availability of data and materials

The datasets used and/or analysed during the current study are available from the corresponding author on reasonable request.

\section{Declarations}

Ethics approval and consent to participate Not applicable.

Consent for publication

Not applicable. 


\section{Competing interests}

The authors declare that they have no competing interests.

Received: 16 March 2021 Accepted: 28 October 2021 Published online: 20 November 2021

\section{References}

1. Gmeinder M, Morgan D, Mueller M. How much do OECD countries spend on prevention? Paris: OECD; 2017. No.101. https://www.oecd-ilibrary.org/ docserver/f19e803c-en.pdf?expires=1615564397\&id=id\&accname= guest\&checksum =6F17AF77FDFC3CEC5B2B00B21E8F8790. Accessed 15 Oct 2021.

2. World Health Organization: About Us; 2020. http://www.emro.who.int/a bout-who/public-health-functions/health-promotion-disease-prevention. html. Accessed 15 Oct 2021

3. Drummond MF, Sculpher MJ, Claxton K, Stoddart GL, Torrance GW. Methods for the economic evaluation of health care programmes. 4th ed. Oxford: Oxford University Press; 2015.

4. Banke-Thomas AO, Madaj B, Charles A, van den Broek N. Social return on investment (SROI) methodology to account for value for money of public health interventions: a systematic review. BMC Public Health. 2015;15(1):582. https://doi.org/10.1186/s12889-015-1935-7.

5. Alayli-Goebbels AF, Evers SM, Alexeeva D, Ament AJ, de Vries NK, Tilly JC, et al. A review of economic evaluations of behavior change interventions: setting an agenda for research methods and practice. J Public Health (Oxf). 2014;36(2):336-44. https://doi.org/10.1093/pubmed/fdt080.

6. Wanless D. Securing Good Health for the Whole Population. London: HM Treasury; 2004. https://commed.vcu.edu/IntroPH/Introduction/wanless_hea Ith_trends.pdf. Accessed 15 Oct 2021.

7. Drummond M, Weatherly H, Claxton K, Cookson R, Ferguson B, Godfrey C, et al. Assessing the challenges of applying standard methods of economic evaluation to public health programmes. York: Public Health Research Consortium; https://www.phrc.online/assets/uploads/files/PHRC_D1_05_Fina I_Report.pdf. Accessed 15 Oct 2021.

8. Briggs AD, Wolstenholme J, Blakely T, Scarborough P. Choosing an epidemiological model structure for the economic evaluation of noncommunicable disease public health interventions. Popul Health Metrics. 2016;14(1):17. https://doi.org/10.1186/s12963-016-0085-1.

9. Cookson $\mathrm{R}$, Drummond $\mathrm{M}$, Weatherly $\mathrm{H}$. Explicit incorporation of equity considerations into economic evaluation of public health interventions. Health Econ Policy Law. 2009;4(Pt 2):231-45. https://doi.org/10.1017/S1 744133109004903

10. Page MJ, McKenzie JE, Bossuyt PM, Boutron I, Hoffmann TC, Mulrow CD, et al. The PRISMA 2020 statement: an updated guideline for reporting systematic reviews. BMJ. 2021;372:n71.

11. Tricco AC, Lillie E, Zarin W, O'Brien K, Colquhoun H, Kastner M, et al. A scoping review on the conduct and reporting of scoping reviews. BMC Med Res Methodol. 2016;16(1):15. https://doi.org/10.1186/s12874-016-0116-4.

12. Shea BJ, Grimshaw JM, Wells GA, Boers M, Andersson N, Hamel C, et al. Development of AMSTAR: a measurement tool to assess the methodological quality of systematic reviews. BMC Med Res Methodol. 2007;7(1):10. https://doi.org/10.1186/1471-2288-7-10.

13. Popay J, Roberts H, Sowden A, Petticrew M, Arai L, Rodgers, M et al. Guidance on the Conduct of Narrative Synthesis in Systematic Reviews. 2006. http://citeseerx.ist.psu.edu/viewdoc/download?doi=10.1.1.178.31 00\&rep=rep1\&type $=$ pdf. Accessed 15 Oct 2021.

14. Edwards RT, Charles JM, Lloyd-Williams H. Public health economics: a systematic review of guidance for the economic evaluation of public health interventions and discussion of key methodological issues. BMC Public Health. 2013;13(1):1001. https://doi.org/10.1186/1471-2458-13-1 001.

15. Honeycutt AA, Clayton L, Khavjou O, Finkelstein E, Prabhu M, Blitstein J, et al. Guide to analyzing the cost-effectiveness of community public health prevention approaches. Office of the Assistant Secretary for Planning and Evaluation; 2006. https://aspe.hhs.gov/pdf-report/guide-ana lyzing-cost-effectiveness-community-public-health-prevention-approaches. Accessed 15 Oct 2020.

16. van Mastrigt GA, Paulus AT, Aarts MJ, Evers SM, Alayli-Goebbels AF. A qualitative study on the views of experts regarding the incorporation of non-health outcomes into the economic evaluations of public health interventions. BMC Public Health. 2015;15(1):954. https://doi.org/10.1186/s12 889-015-2247-7.

17. Benning TM, Alayli-Goebbels AF, Aarts MJ, Stolk E, de Wit GA, Prenger R, et al. Exploring outcomes to consider in economic evaluations of health promotion programs: what broader non-health outcomes matter Most? BMC Health Serv Res. 2015;15(1):266. https://doi.org/10.1186/s12913-015-0908-y.

18. Drost RMW, Paulus ATG, Ruwaard D, Evers SM. Inter-sectoral costs and benefits of mental health prevention: towards a new classification scheme. J Ment Health Policy Econ. 2013;16(4):179-86.

19. Weatherly H, Drummond M, Claxton K, Cookson R, Ferguson B, Godfrey C, et al. Methods for assessing the cost-effectiveness of public health interventions: key challenges and recommendations. Health Policy. 2009; 93(2-3):85-92. https://doi.org/10.1016/j.healthpol.2009.07.012.

20. Cookson R, Mirelman AJ, Griffin S, Asaria M, Dawkins B, Norheim OF, et al. Using cost-effectiveness analysis to address health equity concerns. Value Health. 2017;20(2):206-12. https://doi.org/10.1016/j.jval.2016.11.027.

21. Campbell M, McKenzie JE, Sowden A, Vittal Katikireddi S, Brennan SE, Ellis $S$, et al. Synthesis without meta-analysis (SWiM) in systematic reviews: reporting guideline. BMJ. 2020;368:16890. https://doi.org/10.113 6/bmj.16890.

22. McKenzie JE, Brennan SE. Chapter 12: synthesizing and presenting findings using other methods. In: Higgins JPT, Thomas J, Chandler J, Cumpston M, Li T, Page MJ, Welch VA (editors). Cochrane handbook for systematic reviews of interventions version 6.2 (updated February 2021). Cochrane, 2021. Available from www.training.cochrane.org/handbook.

23. Cochrane M, Watson PM, Timpson H, Haycox A, Collins B, Jones L, et al. Systematic review of the methods used in economic evaluations of targeted physical activity and sedentary behaviour interventions. Soc Sci Med. 2019;232:156-67. https://doi.org/10.1016/j.socscimed.2019.04.040.

24. Reeves P, Edmunds K, Searles A, Wiggers J. Economic evaluations of public health implementation-interventions: a systematic review and guideline for practice. Public Health. 2019;169:101-13. https://doi.org/10.1016/.jpuhe.2019.01.012.

25. Huter K, Dubas-Jakóbczyk K, Kocot E, Kissimova-Skarbek K, Rothgang H. Economic evaluation of health promotion interventions for older people: do applied economic studies meet the methodological challenges? Cost Eff Resour Alloc. 2018;16(1):14. https://doi.org/10.1186/s12962-018-0100-4.

26. Oosterhoff M, Bosma H, van Schayck OCP, Evers S, Dirksen CD, Joore MA. A systematic review on economic evaluations of school-based lifestyle interventions targeting weight-related behaviours among 4-12 year olds: issues and ways forward. Prev Med. 2018;114:115-22. https://doi.org/10.101 6/j.ypmed.2018.06.015.

27. Dubas-Jakóbczyk K, Kocot E, Kissimova-Skarbek K, Huter K, Rothgang H. Economic evaluation of health promotion and primary prevention actions for older people-a systematic review. Eur J Pub Health. 2017;27(4):670-9. https://doi.org/10.1093/eurpub/ckx030.

28. Doring N, Mayer S, Rasmussen F, Sonntag D. Economic Evaluation of Obesity Prevention in Early Childhood: Methods, Limitations and Recommendations. Int J Environ Res Public Health. 2016;13:9. https://doi. org/10.3390/ijerph13090911.

29. Polinder S, Segui-Gomez M, Toet H, Belt E, Sethi D, Racioppi F, et al. Systematic review and quality assessment of economic evaluation studies of injury prevention. Accid Anal Prev. 2012;45:211-21. https://doi.org/10.101 6/j.aap.2011.07.004.

30. Zanganeh M, Adab P, Li B, Frew E. A systematic review of methods, study quality, and results of economic evaluation for childhood and adolescent obesity intervention. Int J Environ Res Public Health. 2019;163(3):485. https:// doi.org/10.3390/ijerph16030485.

31. Hill SR, Vale L, Hunter D, Henderson E, Oluboyede Y. Economic evaluations of alcohol prevention interventions: is the evidence sufficient? A review of methodological challenges. Health Pol. 2017;121(12):1249-62. https://doi. org/10.1016/j.healthpol.2017.10.003.

32. Squires $H$, Chilcott J, Akehurst R, Burr J, Kelly MP. A framework for developing the structure of public health economic models. Value Health. 2016;19(5):588-601. https://doi.org/10.1016/j.jval.2016.02.011.

33. Chalkidou K, Culyer A, Naidoo B, Littlejohns P. Cost-effective public health guidance: asking questions from the decision-maker's viewpoint. Health Econ. 2008;17(3):441-8. https://doi.org/10.1002/hec.1277.

34. Husereau D, Drummond M, Petrou S, Carswell C, Moher D, Greenberg D, et al. Consolidated health economic evaluation reporting standards (CHEERS) statement. Value Health. 2013;16(2):e1-5. https://doi.org/10.1016/j. jval.2013.02.010 
35. Von Bertalanffy L. General system theory: foundations, development, applications. New York: George Brazillier, Inc:; 1968.

36. Dahlgren $\mathrm{G}$, Whitehead M. Policies and strategies to promote social equity in health (mimeo). Stockholm: Institute for Future Studies; 1991. https:// core.ac.uk/download/pdf/6472456.pdf. Accessed 15 Oct 2021.

37. Li G, Sajobi TT, Menon BK, Korngut L, Lowerison M, James M, et al. Registrybased randomised controlled trials- what are the advantages, challenges, and areas for future research? J Clin Epidemiol. 2016;80:16-24. https://doi. org/10.1016/j.jclinepi.2016.08.003.

38. Payne K, McAllister M, Davies LM. Valuing the economic benefits of complex interventions: when maximising health is not sufficient. Health Econ. 2013;22(3):258-71. https://doi.org/10.1002/hec.2795.

39. Lorgelly PK, Lawson KD, Fenwick EA, Briggs AH. Outcome measurement in economic evaluations of public health interventions: a role for the capability approach? Int J Environ Res Public Health. 2010;7(5):2274-89. https://doi. org/10.3390/ijerph7052274.

40. Alayli-Goebbels AF, Dellaert BG, Knox SA, Ament AJ, Lakerveld J, Bot SD, et al. Consumer preferences for health and nonhealth outcomes of health promotion: results from a discrete choice experiment. Value Health. 2013; 16(1):114-23. https://doi.org/10.1016/j.jval.2012.08.2211.

41. Flynn TN, Huynh E, Peters TJ, Al-Janabi H, Clemens S, Moody A, et al. Scoring the Icecap-a capability instrument. Estimation of a UK general population tariff. Health Econ. 2015;24(3):258-69. https://doi.org/10.1002/ hec.3014.

42. Mayer S, Paulus ATG, Laszewska A, Simon J, Drost RMWA, Ruwaard D, et al. Health-related resource-use measurement instruments for Intersectoral costs and benefits in the education and criminal justice sectors. PharmacoEcon. 2017;35(9):895-908. https://doi.org/10.1007/s40273-017-0522-4.

43. Rodriguez-Sanchez B, Aranda-Reneo I, Oliva-Moreno J, Lopez-Bastida J. Assessing the effect of including social costs in economic evaluations of diabetes-related interventions: a systematic review. Clinicoecon Outcomes Res. 2021;13:307-34. https://doi.org/10.2147/CEOR.S301589.

44. Tugwell P, de Savigny D, Hawker G, Robinson V. Applying clinical epidemiological methods to health equity: the equity effectiveness loop. BMJ. 2006:332(7537):358-61. https://doi.org/10.1136/bmj.332.7537.358.

45. Squires H, Chilcott J, Akehurst R, Burr J, Kelly MP. A systematic literature review of the key challenges for developing the structure of public health economic models. Int J Public Health. 2016;61(3):289-98. https://doi.org/10.1 007/s00038-015-0775-7.

46. Al-Janabi H, Flynn TN, Coast J. Development of a self-report measure of capability wellbeing for adults: the ICECAP-A. Qual Life Res. 2012;21(1):16776. https://doi.org/10.1007/s11136-011-9927-2.

\section{Publisher's Note}

Springer Nature remains neutral with regard to jurisdictional claims in published maps and institutional affiliations.

Ready to submit your research? Choose BMC and benefit from:

- fast, convenient online submission

- thorough peer review by experienced researchers in your field

- rapid publication on acceptance

- support for research data, including large and complex data types

- gold Open Access which fosters wider collaboration and increased citations

- maximum visibility for your research: over $100 \mathrm{M}$ website views per year

At $\mathrm{BMC}$, research is always in progress.

Learn more biomedcentral.com/submissions 